\title{
Exact Periodic Wave Solutions of Two Types of Modified Boussinesq Equations*
}

\author{
Y.-Z. PENG ${ }^{\dagger}$
}

Key Laboratory of Pure and Applied Mathematics School of Mathematical Sciences, Peking University Beijing 100871, P.R. China

(Received September 30, 2002; revised version February 24, 2003)

Exact periodic wave solutions to two types of modified Boussinesq equations are obtained by the use of the Jacobi elliptic function method in a unified form. Some new, general solitary wave solutions are presented.

PACS numbers: 02.30.Jr

\section{Introduction}

The travelling wave solution, meaning a solution of constant form moving with a fixed velocity, is one of the fundamental objects in the study of equations modelling wave phenomena. Of particular interest are three types of travelling waves: the solitary waves, which are localized travelling waves, asymptotically zero at large distances, the periodic waves, and the kink waves, which rise or descend from one asymptotic state to another. Recently, the periodic wave solutions to nonlinear evolution equations has attracted considerable interest [1-6]. Porubov et al. [1-3] have obtained some exact periodic wave solutions to some nonlinear wave equations in terms of the Weierstrass elliptic function. However, their method involves complicated deducing. Liu et al. [4, 5] proposed the Jacobi elliptic function method for finding periodic wave solutions to nonlinear evolution equations, but this method involves tedious calculation. In this paper, we use the Jacobi elliptic function method in a unified form to find exact periodic wave solutions to two types of modified Boussinesq equations. Some new solitary wave solutions are presented.

*This work was supported by the Postdoctoral Science Foundation of China.

†e-mail: yanzepeng@163.com 


\section{Periodic wave solutions to the first type of modified Boussinesq equations}

The first type of modified Boussinesq equations [7] is

$$
H_{t}+(H u)_{x}+u_{x x x}=0, \quad u_{t}+H_{x}+u u_{x}=0,
$$

where the subscripts denote partial derivatives. We seek for its travelling wave solution of the form

$$
H(x, t)=H(\xi), \quad u(x, t)=u(\xi), \quad \xi=k x-\omega t .
$$

Without loss of generality, we define $k>0$. Substituting Eq. (2) into Eq. (1) and integrating once, we obtain

$$
-\omega H+k(H u)^{\prime}+k^{3} u^{\prime \prime}=C_{1}, \quad-\omega u+k H+\frac{1}{2} k u^{2}=C_{2},
$$

where the prime denotes the derivative with respect to $\xi$ (throughout the paper), $C_{1}$ and $C_{2}$ - integral constants. We assume that Eq. (3) has solution in the form

$$
H=A_{0}+A_{1} f+A_{2} f^{2}, \quad u=B_{0}+B_{1} f,
$$

with $f$ satisfying the following equation:

$$
f^{\prime \prime}=p f+q f^{3}, \quad f^{\prime 2}=p f^{2}+\frac{1}{2} q f^{4}+r,
$$

where $p, q$, and $r$ are constants to be determined.

Remark. Generally, $H, u$ should be expanded into $\sum_{i=0}^{l} A_{i} f^{i}$ and $\sum_{i=0}^{n} B_{i} f^{i}$, respectively, where $l$ and $n$ are determined by balancing the highest order of derivative term and nonlinear term in Eq. (1), that is $l=2$, and $n=1$. Thus we obtain Eq. (4). The use of Eq. (5) is the key of our method. If we use the Jacobi elliptic function method $[4,5]$, in Eq. (4) $f$ should be replaced by $\operatorname{sn} \xi, \operatorname{cn} \xi, \operatorname{dn} \xi$, and $\operatorname{cs} \xi$, respectively.

The substitution of Eq. (4) into Eq. (3) and use of Eq. (5) yields (equate coefficients of like powers of $f$ to zero)

$$
\begin{aligned}
& A_{2} B_{1}+q k^{2} B_{1}=0, \quad-\omega A_{2}+k\left(A_{1} B_{1}+A_{2} B_{0}\right)=0, \\
& -\omega A_{1}+k\left(A_{0} B_{1}+A_{1} B_{0}\right)+p k^{3} B_{1}=0, \quad-\omega A_{0}+k A_{0} B_{0}=C_{1}, \\
& k A_{2}+\frac{1}{2} k B_{1}^{2}=0, \quad-\omega B_{1}+k A_{1}+k B_{0} B_{1}=0, \\
& -\omega B_{0}+k A_{0}+\frac{1}{2} k B_{0}^{2}=C_{2},
\end{aligned}
$$

from which we have

$$
A_{0}=-p k^{2}, \quad A_{1}=0, \quad A_{2}=-q k^{2}, \quad B_{0}=\frac{\omega}{k}, \quad B_{1}= \pm \sqrt{2 q} k,
$$

which demands $q \geq 0$ 
Therefore the exact solution of Eq. (1) reads

$$
H=-p k^{2}-q k^{2} f^{2}, \quad u=\frac{\omega}{k} \pm \sqrt{2 q} k f,
$$

with $f$ satisfying Eq. (5).

As examples, we discuss the following two cases.

Case 1. $p=-\left(1+m^{2}\right), q=2 m^{2}, r=1$.

In this case, Eq. (5) has solution $f=\operatorname{sn} \xi$, so we obtain periodic wave solution to Eq. (1)

$$
H=\left(1+m^{2}\right) k^{2}-2 m^{2} k^{2} \operatorname{sn}^{2}(k x-\omega t), \quad u=\frac{\omega}{k} \pm 2 m k \operatorname{sn}(k x-\omega t) .
$$

As $m \rightarrow 1, \operatorname{sn} \xi \rightarrow \tanh \xi$, and we get solitary wave solution of Eq. (1)

$$
H=2 k^{2} \operatorname{sech}^{2}(k x-\omega t), \quad u=\frac{\omega}{k} \pm 2 k \tanh (k x-\omega t) .
$$

Case 2. $p=2-m^{2}, q=2, r=m^{\prime 2} \equiv 1-m^{2}$.

Now the solution of Eq. (5) reads $f=\operatorname{cs} \xi \equiv \operatorname{cn} \xi / \operatorname{sn} \xi$. Thus another periodic wave solution to $\mathrm{Eq}$. (1) is

$$
H=-\left(2-m^{2}\right) k^{2}-2 k^{2} \operatorname{cs}^{2}(k x-\omega t), \quad u=\frac{\omega}{k} \pm 2 k \operatorname{cs}(k x-\omega t) .
$$

For $m \rightarrow 1, \operatorname{cs} \xi \rightarrow \operatorname{csch} \xi$, Eq. (11) degenerates as

$$
H=-k^{2}-2 k^{2} \operatorname{csch}^{2}(k x-\omega t), \quad u=\frac{\omega}{k} \pm 2 k \operatorname{csch}(k x-\omega t),
$$

which is a new solitary wave solution (singular) of Eq. (1). Because $q \geq 0$ in Eq. (8), Eq. (1) does not admit cn- and dn-function wave solutions of polynomial form. Let us notice that $\operatorname{sn} \xi, \operatorname{cn} \xi$, and $\operatorname{dn} \xi$ are the Jacobi elliptic sine function, cosine function, and the third kind of the Jacobi elliptic function, respectively, and $m$ is the modulus of the Jacobi elliptic functions, about which detailed discussion can be found in $[8-10]$.

\section{Periodic wave solutions to the second type of modified Boussinesq equations}

The second type of modified Boussinesq equation [11] is

$$
h_{t}+u_{x}+(h u)_{x}-\alpha u_{x x x}=0, \quad u_{t}+u u_{x}+h_{x}-3 \alpha u_{x x t}=0 .
$$

We suppose that Eq. (13) has solution of the form

$$
\begin{aligned}
& h(x, t) \equiv h(\xi)=A_{0}+A_{1} f+A_{2} f^{2}, \\
& u(x, t) \equiv u(\xi)=B_{0}+B_{1} f+B_{2} f^{2},
\end{aligned}
$$

with $f$ satisfying Eq. (5). Substituting Eq. (14) into Eq. (13) and using Eq. (5), with the same procedure as in Sec. 2, we obtain 


$$
\begin{aligned}
& A_{0}=-1+\frac{k^{2}}{36 \omega^{2}}+2 p \alpha k^{2}, \quad A_{1}=0, \quad A_{2}=3 q \alpha k^{2}, \\
& B_{0}=\frac{\omega}{k}+\frac{k}{6 \omega}-12 p \alpha \omega k, \quad B_{1}=0, \quad B_{2}=-18 q \alpha \omega k .
\end{aligned}
$$

Therefore the exact solution of Eq. (13) is

$$
\begin{aligned}
& h=-1+\frac{k^{2}}{36 \omega^{2}}+2 p \alpha k^{2}+3 q \alpha k^{2} f^{2}, \\
& u=\frac{\omega}{k}+\frac{k}{6 \omega}-12 p \alpha \omega k-18 q \alpha \omega k f^{2},
\end{aligned}
$$

where $f$ satisfies Eq. (5).

In what follows several cases are discussed.

Case 1. $p=-\left(1+m^{2}\right), q=2 m^{2}, r=1$.

As case 1 in Sec. 2, we have periodic solution to Eq. (13)

$$
\begin{aligned}
& h=-1+\frac{k^{2}}{36 \omega^{2}}-2\left(1+m^{2}\right) \alpha k^{2}+6 m^{2} \alpha k^{2} \operatorname{sn}^{2}(k x-\omega t), \\
& u=\frac{\omega}{k}+\frac{k}{6 \omega}+12\left(1+m^{2}\right) \alpha \omega k-36 m^{2} \alpha \omega k \operatorname{sn}^{2}(k x-\omega t) .
\end{aligned}
$$

Its corresponding general solitary wave solution is

$$
\begin{aligned}
& h=-1+\frac{k^{2}}{36 \omega^{2}}-4 \alpha k^{2}+6 \alpha k^{2} \tanh ^{2}(k x-\omega t), \\
& u=\frac{\omega}{k}+\frac{k}{6 \omega}+24 \alpha \omega k-36 \alpha \omega k \tanh ^{2}(k x-\omega t) .
\end{aligned}
$$

Case 2. $p=2 m^{2}-1, q=-2 m^{2}, r=m^{\prime 2} \equiv 1-m^{2}$.

From Eq. (5) we have $f=c n \xi$, and Eq. (13) has periodic wave solution

$$
\begin{aligned}
& h=-1+\frac{k^{2}}{36 \omega^{2}}+2\left(2 m^{2}-1\right) \alpha k^{2}-6 m^{2} \alpha k^{2} \operatorname{cn}^{2}(k x-\omega t), \\
& u=\frac{\omega}{k}+\frac{k}{6 \omega}-12\left(2 m^{2}-1\right) \alpha \omega k+36 m^{2} \alpha \omega k \mathrm{cn}^{2}(k x-\omega t) .
\end{aligned}
$$

For $m \rightarrow 1, \operatorname{cn} \xi \rightarrow \operatorname{sech} \xi$, and Eq. (19) degenerates as Eq. (18).

Case 3. $p=2-m^{2}, q=-2, r=-m^{2}$.

The solution of Eq. (5) reads $f=\operatorname{dn} \xi$. Therefore we get another periodic wave solution of Eq. (13)

$$
\begin{aligned}
& h=-1+\frac{k^{2}}{36 \omega^{2}}+2\left(2-m^{2}\right) \alpha k^{2}-6 \alpha k^{2} \operatorname{dn}^{2}(k x-\omega t), \\
& u=\frac{\omega}{k}+\frac{k}{6 \omega}-12\left(2-m^{2}\right) \alpha \omega k+36 \alpha \omega k \operatorname{dn}^{2}(k x-\omega t) .
\end{aligned}
$$

As $m \rightarrow 1, \operatorname{dn} \xi \rightarrow \operatorname{sech} \xi$, and we obtain the solution to Eq. (18) again. 
Case 4. $p=2-m^{2}, q=2, r=m^{\prime 2}$.

As case 2 in Sec. 2, we get periodic wave solution to Eq. (13)

$$
\begin{aligned}
& h=-1+\frac{k^{2}}{36 \omega^{2}}+2\left(2-m^{2}\right) \alpha k^{2}+6 \alpha k^{2} \operatorname{cs}^{2}(k x-\omega t), \\
& u=\frac{\omega}{k}+\frac{k}{6 \omega}-12\left(2-m^{2}\right) \alpha \omega k-36 \alpha \omega k \operatorname{cs}^{2}(k x-\omega t) .
\end{aligned}
$$

As $m \rightarrow 1$, we obtain a new solitary wave solution of Eq. (13)

$$
\begin{aligned}
& h=-1+\frac{k^{2}}{36 \omega^{2}}+2 \alpha k^{2}+6 \alpha k^{2} \operatorname{csch}^{2}(k x-\omega t), \\
& u=\frac{\omega}{k}+\frac{k}{6 \omega}-12 \alpha \omega k-36 \alpha \omega k \operatorname{csch}^{2}(k x-\omega t) .
\end{aligned}
$$

\section{Conclusion}

We have obtained exact periodic wave solutions of two types of modified Boussinesq equations by means of the Jacobi elliptic function method in a unified form. In contrast to the Jacobi elliptic function method, some merits are obviously a vailable for our method, which is applicable to a large variety of nonlinear partial differential equations, as long as the odd- and even-order derivative terms do not coexist in the equation under consideration. It is shown that this method is more general than the hyperbolic tangent function method. In fact we may obtain more Jacobi elliptic wave solutions through Eq. (5). Due to the limitation of space, we do not discuss it.

\section{References}

[1] A.V. Porubov, Phys. Lett. A 221, 391 (1996).

[2] A.V. Porubov, M.G. Velarde, J. Math. Phys. 40, 884 (1999).

[3] A.V. Porubov, D.F. Parker, Wave Motion 29, 97 (1999).

[4] S.K. Liu, Z. Fu, S.D. Liu, Q. Zhao, Phys. Lett. A 289, 69 (2001).

[5] Z. Fu, S.K. Liu, S.D. Liu, Q. Zhao, Phys. Lett. A 290, 72 (2001).

[6] E.J. Parkes, B.R. Duffy, P.C. Abbott, Phys. Lett. A 295, 280 (2002).

[7] R.L. Sachs, Physica D 30, 1 (1988).

[8] F. Bowman, Introduction to Elliptic Functions with Applications, Universities, London 1959.

[9] S.K. Liu, S.D. Liu, Nonlinear Equations in Physics, Peking University Press, Beijing 2000 (in Chinese).

[10] V. Prasolov, Y. Solovyev, Elliptic Functions and Elliptic Integrals, American Mathematical Society, Providence 1997.

[11] G.B. Whitham, Linear and Nonlinear Waves, Wiley, New York 1973. 